\title{
Gene set enrichment analysis of pathways and transcription factors associated with diabetic retinopathy using a microarray dataset
}

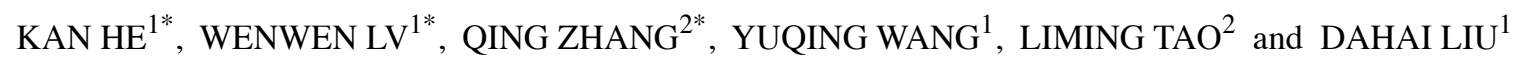 \\ ${ }^{1}$ Center for Stem Cell and Translational Medicine, School of Life Sciences, Anhui University; \\ ${ }^{2}$ Department of Ophthalmology, The Second Hospital of Anhui Medical University, Hefei, Anhui 230601, P.R. China
}

Received March 4, 2015; Accepted May 12, 2015

DOI: $10.3892 / \mathrm{ijmm} .2015 .2220$

\begin{abstract}
Diabetic retinopathy (DR) is a serious microvascular complication of diabetes, which causes visual disability and blindness. Several studies have used gene expression profiling of DR to identify the key genes involved in this process; however, few studies have focused on the associated pathways and transcription factors (TFs), or on the co-expression patterns at the multiple pathways level. In this study, we employed a microarray dataset from the public database library of the Gene Expression Omnibus (GEO) associated with DR and applied gene set enrichment analysis (GSEA) to this dataset and performed candidate TF selection. As a result, 10 upregulated pathways, including the type I diabetes mellitus and peroxisome proliferator-activated receptor (PPAR) signaling pathways, as well as 59 downregulated pathways, including the ErbB signaling pathway and the mammalian target of rapamycin (mTOR) signaling pathway, were identified as DR-related pathways. The majority of these pathways have been previously identified, but some were novel. Finally, co-expression networks of related pathways were constructed using the significant core genes and TFs, such as PPAR $\gamma$ and SMAD4. The results of our study may enhance our understanding of the molecular mechanisms associated DR at the genome-wide level.
\end{abstract}

Correspondence to: Professor Dahai Liu, Center for Stem Cell and Translational Medicine, School of Life Sciences, Anhui University, 111 Jiulong Road, Hefei, Anhui 230601, P.R. China

E-mail: seansean2014@126.com

Professor Liming Tao, Department of Ophthalmology, The Second Hospital of Anhui Medical University, 678 Furong Road, Hefei, Anhui 230601, P.R. China

E-mail:1mtao9@163.com

*Contributed equally

Key words: diabetic retinopathy, pathway, gene set enrichment analysis, peroxisome proliferator-activated receptor, SMAD

\section{Introduction}

Diabetic retinopathy (DR), a specific microvascular complication of diabetes, is the most common cause of visual disability and blindness. The prevalence of DR increases with the duration of diabetes (1), and nearly $99 \%$ of patients with type 1 diabetes and $60 \%$ with type 2 have some degree of DR after 20 years $(1,2)$. DR can be classified into 2 stages: non-proliferative and proliferative. The earliest visible signs of non-proliferative DR are microaneurysms, hemorrhages, hard exudates, cotton wool spots, intraretinal microvascular abnormalities and venous beading. The more severe state of proliferative DR (PDR) is characterized by the growth of new blood vessels on the surface of the retina or the optic disc, which are prone to hemorrhaging. Finally, visual impairment results in vitreous hemorrhage, subsequent fibrosis and tractional retinal detachment $(3,4)$. Although the pathogenesis of DR has not yet been fully elucidated, the pathogenesis of diabetes is believed to be multifactorial, with genetic risk factors playing a fundamental role. However, several factors, including hyperglycemia, aldose reductase, advanced glycation end products (AGEs) and cytokines, such as vascular endothelial growth factor (VEGF) have been implicated in the disease pathogenesis (5).

Despite DR being a common complication of diabetes, little is known about the underlying molecular mechanisms. In recent years, the complex association that exists between the most relevant contributors to the onset and progression of DR, such as AGEs, oxidative stress, inflammation and angiogenesis have been elucidated and analyzed, particularly via whole-genome expression analyses using cells and animal models $(6,7)$. Based on published studies, several systems, pathways and processes have been strongly implicated in DR; these include the renin-angiotensin system, the polyol pathway, non-enzymatic glycation, endothelial dysfunction, the maintenance of vascular tone, extracellular matrix remodeling and angiogenesis, which is dysregulated in diabetes and leads to the proliferation of new, fragile retinal capillaries and culminates in $\operatorname{PDR}(8,9)$. A host of genes involved in these pathways/processes have been treated as potential candidate genes. These genes include angiotensin I-converting enzyme (ACE), angiotensin II type 1 receptor (AGTR1), angiotensinogen (AGT), VEGF, aldose reductase (AR2), receptor for advanced glycation end products (RAGE), glucose trans- 
porter 1 (GLUT1), inducible nitric oxide synthase (NOS2A), constitutive nitric oxide synthase (NOS3), transforming growth factor- $\beta$ (TGF- $\beta$ ), endothelin isoforms and their cellular receptors, amongst others (10-19). However, to the best of our knowledge, few studies to date have focused on the associated pathways and transcription factors (TFs), or on the co-expression patterns at the multiple pathways level.

In the present study, we employed a microarray dataset of genome-wide gene expression profiling from the Gene Expression Omnibus (GEO; http://www.ncbi.nlm.nih.gov/ geo/) $(20,21)$, which is associated with DR. The most wellknown method of gene set enrichment analysis (GSEA) was used to analyze the genomic data in order to uncover the regulatory mechanisms of retinopathy (damage to the retina) caused by diabetic complications at the multiple pathways level. GSEA is widely used to analyze gene expression profiles, particularly to identify pre-defined gene sets which exhibit significant differences in expression between samples from the control and treatment groups (22-24). The goal of GSEA is to determine other interesting categories (pathways) in which the constituent genes exhibit coordinated changes in expression under the given experimental conditions, other than in the form of sets of differentially expressed genes (DEGs). One of the advantages of GSEA is that it has the ability to highlight genes that are weakly connected to the phenotype through pathway analysis, something which may be difficult to detect using classical univariate statistics (22).

\section{Materials and methods}

Microarray data collection and pre-processing. We searched the GEO database(www.ncbi.nlm.nih.gov/geo/) for gene expression profiling studies associated with DR. Data were included in our re-analysis if they met the following conditions: i) the data were genome-wide; ii) a comparison was conducted between DR samples and control (CT) samples; and iii) complete microarray raw or normalized data were available. Finally, we chose the GSE12610 dataset for our re-analysis, which was contributed by Liang et al (http://www.ncbi.nlm.nih.gov/geo/ query/acc.cgi?acc=GSE12610). In this dataset, a total of 5 RNA samples extracted from retinas were examined for RNA quality and then hybridized to 2 different GeneChip ${ }^{\circledR}$ Mouse Genome 4302.0 arrays (technical replicates; Affymetrix, Santa Clara, CA, USA). There were 3 biological replicates for DR (the samples from GSM315892 to GSM315894, designated as DR-1, DR-2 and DR-3) and 2 for CT (the samples GSM315895 and GSM315896, designated as CT-1 and CT-2).

In order to determine the influence of pre-processing on the comparison, data pre-processing was performed using software packages developed in version 2.6.0 of Bioconductor and $\mathrm{R}$ version 2.10.1. Each Affymetrix dataset was background adjusted, normalized, and $\log 2$ probe-set intensities were calculated using the Robust Multichip Average (RMA) algorithm from the affy package (25).

GSEA. Our GSEA of pathways and genes was performed using the Category package in version 2.6.0 of Bioconductor (26). The goal of GSEA is to determine whether the members of a gene set $\mathrm{S}$ are randomly distributed throughout the entire reference gene list L or are primarily found at the top or bottom. One of the advantages of GSEA is its relative robustness in the face of noise and outliers in the data. In our analysis, the gene sets represented by $<10$ genes were excluded. The t-statistic mean of the genes was computed in each Kyoto Encyclopedia of Genes and Genomes (KEGG) pathway. Using a permutation test with 1,000 repetitions, the cut-off of significance level P-values was chosen as 0.05 for the significant pathways associated with DR. Accordingly, the significant pathways and genes were then identified by comparing the samples with DR and those with no DR. The following classification of identified pathways was based on the pathway maps br08901 of BRITE Functional Hierarchies in the KEGG database (http://www.genome.jp/ kegg-bin/get_htext?br08901.keg). The annotation of significant genes in each pathway was performed using the biomaRt package, BioMart v 0.8 rc3 (version of 0.8 release candidate 3; http://www.biomart.org/). Next, clustering of groups and genes was performed based on the expression of the identified genes in each significant pathway, using the hierarchical clustering method and Pearson's correlation co-efficient.

Regulatory elements (REs) and TFs of co-regulated genes. We used a web server known as the DiRE (distant regulatory elements of co-expressed genes, http://dire.dcode.org/), which uses the Enhancer Identification (EI) method, to predict common REs for our input genes that have co-function in each identified, significantly related pathway (27). It predicts function-specific REs that consist of clusters of specifically associated transcription factor binding sites (TFBSs), and it also scores the association of individual TFs with the biological function shared by the group of input genes. We selected a random set of 5,000 genes in the genome of Mus musculus 9 (mm9) as the background genes. As a result, our predicted TFs have two major parameters, including TF occurrence (the percentage of candidate REs containing a conserved binding site for a particular TF) and TF importance (the product of TF occurrence and TF weight). From our candidate associated TFs with input gene sets, we selected the cut-off value of TF importance as $>0.05$.

\section{Results and Discussion}

Identification of significant pathways associated with DR. Compared to the approach of DEGs, the strategy of GSEA that we used in this study is likely to be more powerful than conventional single-gene methods in the study of complex diseases, in which many genes make subtle contributions. According to our GSEA of the dataset of 5 samples, achieved by comparing the DR to the CT samples, there were 69 significant pathways associated with DR, whose P-values were $<0.05$, including 10 upregulated and 59 downregulated pathways. The coregulated pathways network is highlighted in Fig. 1 (red text indicates upregulated pathways, and green text indicates downregulated pathways). Furthermore, the details of significant genes in these 69 pathways related to DR are available upon request, as is the information on probe set ID and gene symbol. Among these 69 pathways associated with DR, the samples were classified and divided into DR and CT groups by clustering. For example, based on the expression of 86 significant genes whose significance level P-value was $<0.05$ in the downregulated ErbB signaling pathway, which may be clustered into 7 groups of gene 


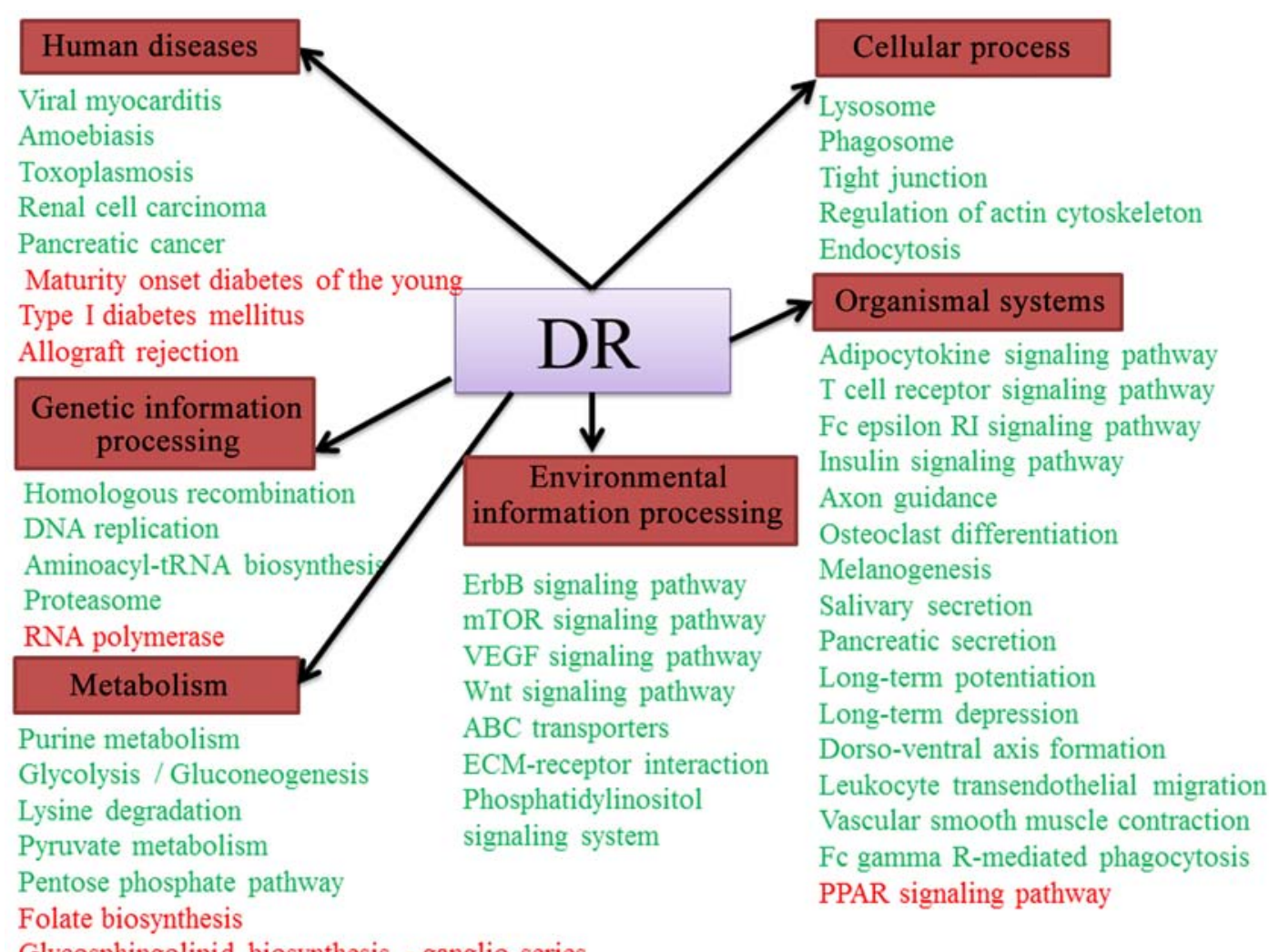

Figure 1. The coregulated pathways network related to diabetic retinopathy (DR) was established based on the 48 significant pathways identified by gene set enrichment analysis (GSEA). The names in red boxes represent 6 Kyoto Encyclopedia of Genes and Genomes (KEGG) pathway maps, and the names of significant pathways (upregulated pathways shown in red text and downregulated pathways shown in green) associated with DR.

sets (Fig. 2; group A-G), 5 samples were clustered into 2 groups, with DR-1, DR-2 and DR-3 in one group and CT-1 and CT-2 in the other group. Similarly, in the downregulated mammalian target of rapamycin (mTOR) pathway, 5 samples were also grouped, as CT-1 and CT-2 in the CT group and DR-1, DR-2 and DR-3 in the DR group (Fig. 3). The 52 genes involved in the mTOR signaling pathway may also be clustered into 4 groups of gene sets (Fig. 3, group A-D). Moreover, in the upregulated peroxisome proliferator-activated receptor (PPAR) pathway, 5 samples were also clustered into 2 groups, with CT-1 and CT- 2 in one group and DR-1, DR-2 and DR-3 in the other group (Fig. 4). Furthermore, 71 genes were involved in the PPAR signaling pathway associated with DR, which may be clustered into 6 groups of gene sets (Fig. 4, group A-F). Moreover, based on the KEGG pathway maps in the KEGG database (http://www. genome.jp/kegg/), the 69 significant pathways could be mapped into 6 functional classes: cellular processes, environmental information processing, genetic information processing, human diseases, metabolism and organismal systems. The details of the pathways involved in each class are described in Tables I-V.

In the functional class of cellular processes, there were 6 significantly downregulated pathways related to DR (Table I). These pathways were involved in cell communication, cell motility, and transport and catabolism. Of these pathways, the tight junction one was the most significant, and was classified as belonging to the functional group of cell communication. As is well known, the breakdown of the blood-retinal barrier (BRB) is one of the most important characteristics of DR and is largely attributed to the disruption of endothelial tight junction. X-box binding protein 1 (XBP1), which is a major transcription factor activated by ER stress, plays an important role in maintaining endothelial barrier function (28). The activation of XBP1 protects against ER stress-induced tight junction damage.

There were 7 significantly downregulated pathways in the functional class of environmental information processing, 5 significantly downregulated pathways in the functional class of genetic information processing, and only 2 significantly upregulated pathways in the functional class of genetic information processing related toDR(Table II). The ATP-binding cassette $(\mathrm{ABC})$ transporters pathway was associated with the function of membrane transport, and the extracellular matrix (ECM)-receptor interaction pathway was associated with the function of the signaling of molecules and interaction. The environmental information processing pathways, such as the ErbB, mTOR, VEGF and Wnt signaling pathways, and the phosphatidylinositol signaling system were related to the functions of signal transduction. The genetic information processing pathways, such as soluble N-ethylmaleimidesensitive attachment protein receptor (SNARE) interactions in vesicular transport and proteasome were related to the functions of folding, sorting and degradation. The genetic information processing pathways of homologous recombination and DNA replication were related to the functions of replication and repair, and. Tthe genetic information processing pathways of aminoacyl-tRNA biosynthesis and ribosome were related to the functions of translation, and the RNA polymerase pathway 


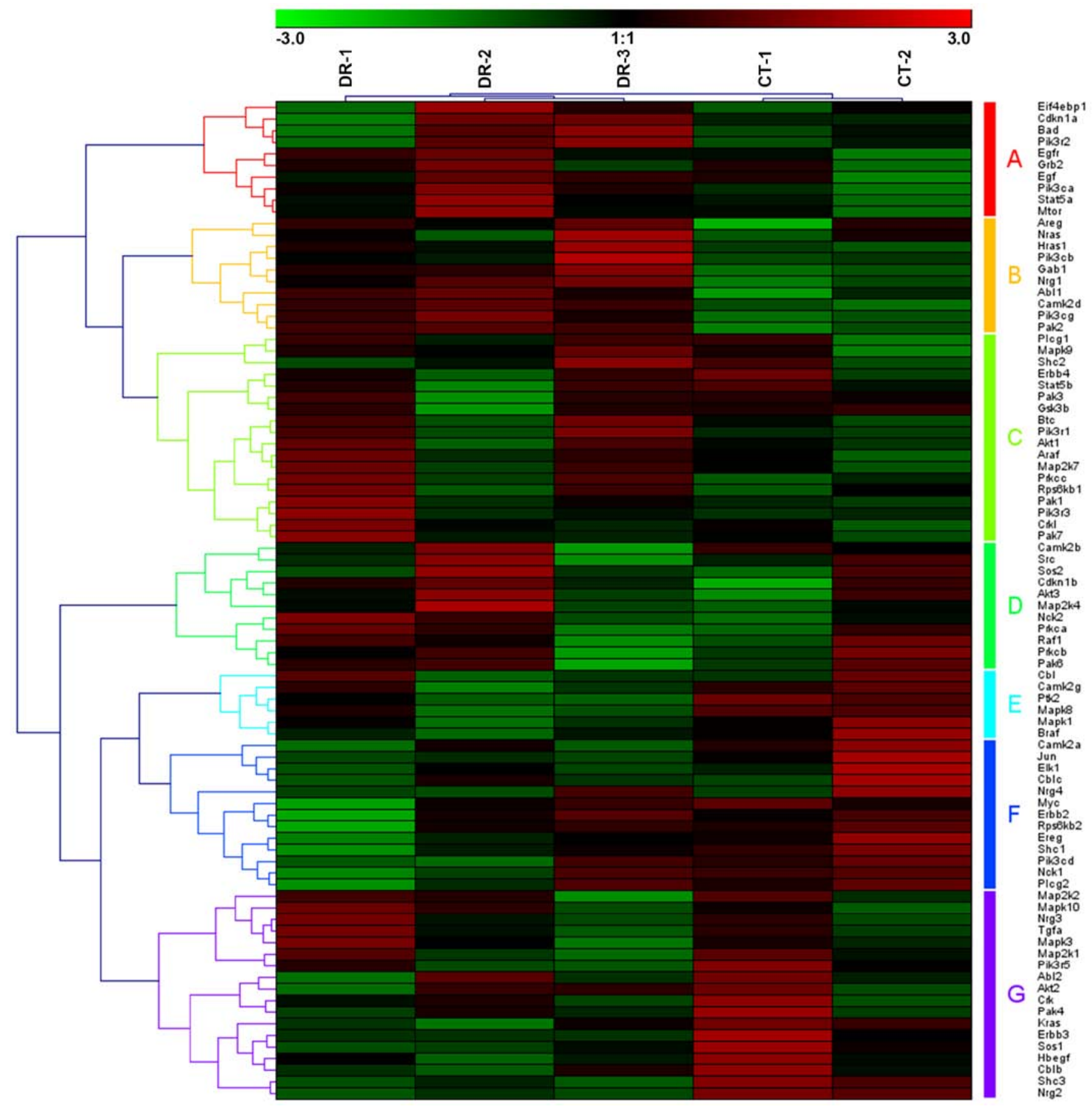

Figure 2. Heatmap of the downregulated ErbB signaling pathway was onstructed using the expression of 86 significant genes (probe sets), which may be clustered into 7 groups of gene sets based on hierarchical clustering with Pearson's correlation co-efficient (group A-G). Five samples were clustered into 2 groups, diabetic retinopathy (DR)-1, DR-2 and DR-3 in one group and control (CT)-1 and CT-2 in the other.

was transcription-related. Of these aforementioned pathways, the ErbB and mTOR signaling pathways were the most significant in this class (the functional class of environmental and genetic information processing). The epidermal growth factor receptor (EGFR), also known as ErbB1/human epidermal growth factor receptor 1 (HER1), is a member of the ErbB family of receptor tyrosine kinases which also includes ErbB2 (Neu, HER2), ErbB3 (HER3) and ErbB4 (HER4). It was recently observed that hyperglycemia perturbs the EGFR-PI3K-AKT and extracellular signal-regulated kinase (ERK) signaling pathways in normal and healing corneas and that increased levels of cellular apoptosis and decreased cell proliferation may be contributing factors in the impairment of corneal epithelial wound healing in diabetic corneas $(29,30)$. Signaling through the mTOR pathway plays a major role in smooth muscle and endothelial cell proliferation in response to hypoxia (31). There have been signs that the inhibition of the PI3K/Akt/mTOR pathway may have beneficial therapeutic effects in the management of PDR, which stems from findings that indicate that growth factors known to play major roles in the induction of angiogenesis depend on 


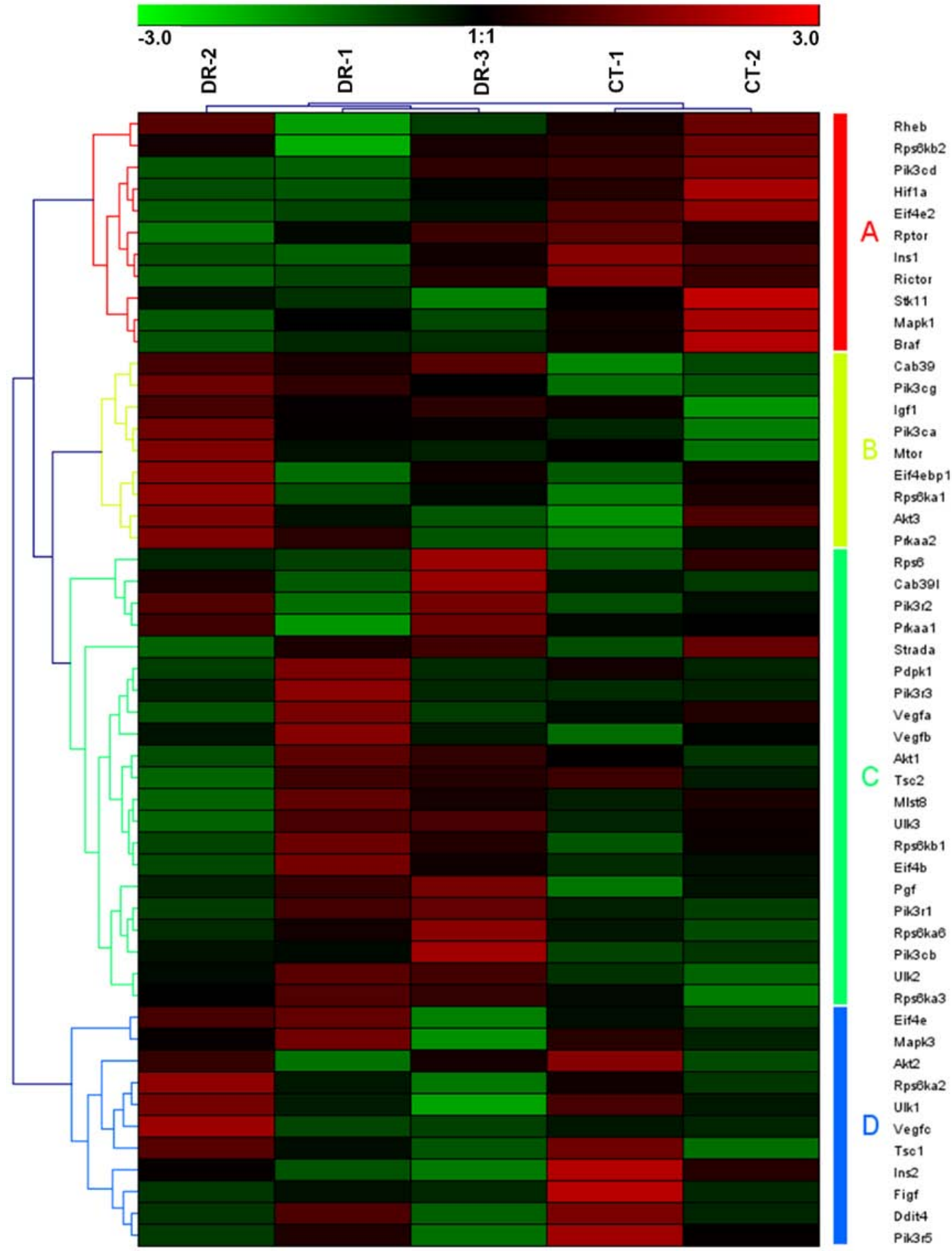

Figure 3. Heatmap of the downregulated of mTOR signaling pathway was constructed using the expression of 52 significant genes (probe sets), which may be clustered into 4 groups of gene sets based on hierarchical clustering with Pearson's correlation co-efficient (group A-D). Five samples were grouped, with CT-1 and CT-2 in the control (CT) group and diabetic retinopathy (DR)-1, DR-2 and DR-3 in the DR group.

PI3K/Akt/mTOR for prolonging the cell survival signals that are operating in pathological angiogenesis (32).

Thirteen significantly associated pathways were classified and assigned to the functional class of human diseases, including 2 upregulated endocrine and metabolic diseases related pathways, 1 upregulated immune diseases related pathways, 3 downregulated cardiovascular diseases related pathways, 1 downregulated infectious diseases: bacterial related pathways, 4 downregulated infectious diseases: parasitic related pathways and 2 downregulated cancer related pathways (Table III). Of these, the pathway of type I diabetes mellitus was one of the most significant endocrine and metabolic diseases-related pathways. The prevalence of DR increases with the duration of diabetes. After 20 years of diabetes, nearly all patients with type I diabetes and $>60 \%$ of patients with type II diabetes have some degree of retinopathy (33).

In the functional class of metabolism, there were 14 downregulated and 4 upregulated significant pathways associated 


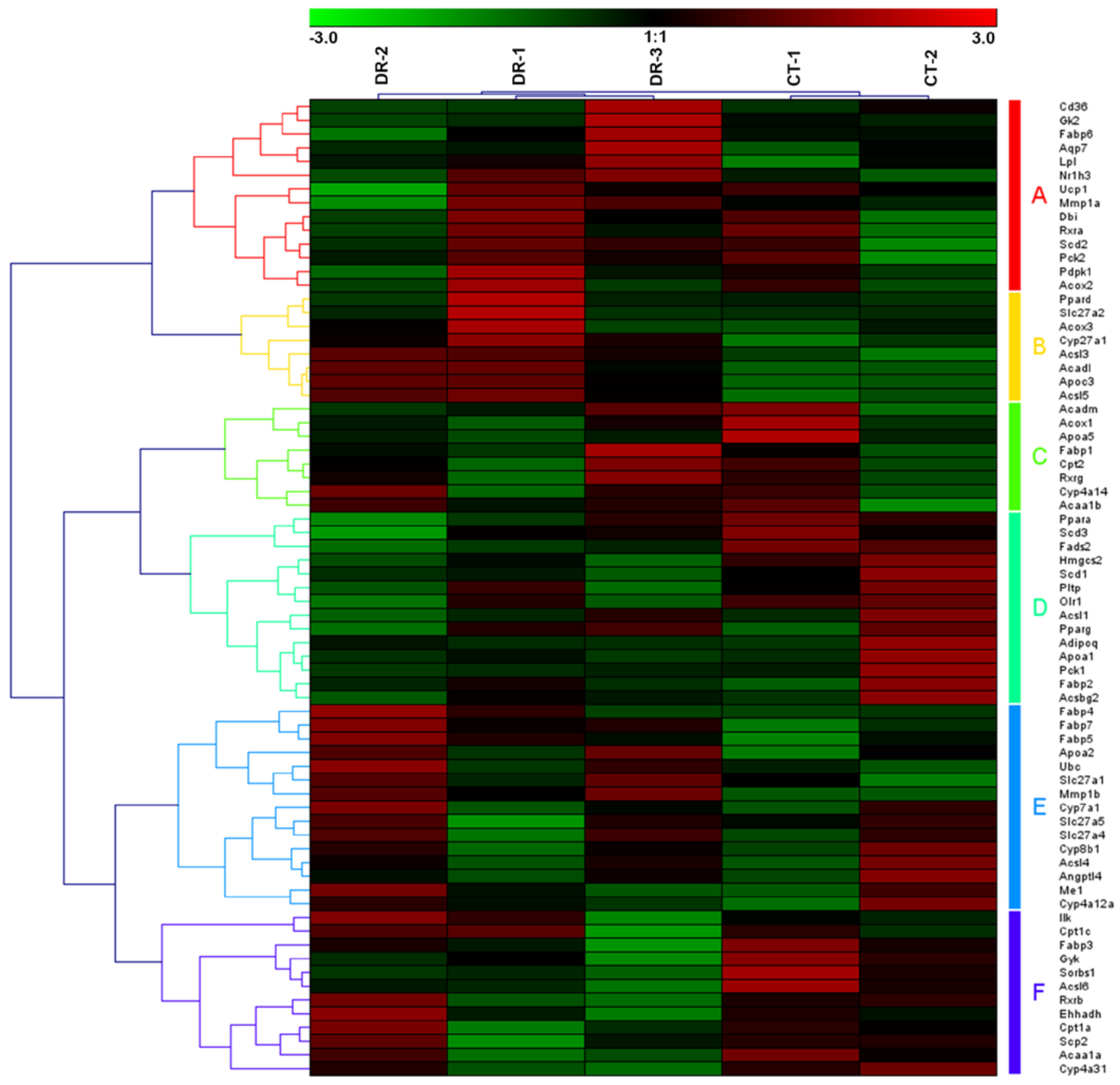

Figure 4. Heatmap of the upregulated peroxisome proliferator-activated receptor (PPAR) signaling pathway was constructed using the expression of 71 significant genes (probe sets), which may be clustered into 6 groups of gene sets based on hierarchical clustering with Pearson's correlation co-efficient (group A-F). Five samples were clustered into 2 groups, with control (CT)-1 and CT-2 in one group and diabetic retinopathy (DR)-1, DR-2 and DR-3 in the other group.

with DR (Table IV). These were involved in 7 different types of metabolism, including carbohydrate metabolism, amino acid metabolism, nucleotide metabolism, lipid metabolism, metabolism of co-factors and vitamins, metabolism of terpenoids and polyketides, and glycan biosynthesis and metabolism. Of these, the glycolysis/gluconeogenesis pathway was one of the most significant pathways, which was classified and assigned to the functional group of carbohydrate metabolism. Protein kinase $\mathrm{C}(\mathrm{PKC})$ is a serine/threonine kinase, which is involved in signal transduction events with regard to specific hormonal, neuronal and growth factor stimuli. Hyperglycaemia leads to an increase in glucose flux through the glycolysis pathway, which in turn increases the de novo synthesis of diacylglycerol (DAG), the key activator of PKC in physiology (34). PKC is a molecule which plays an important role in the regulation of numerous physiological processes, whose upregulation contributes to the pathogenesis of DR.

In the last functional class of organismal systems, there was 1 significantly upregulated and 17 significantly downregulated pathways associated with DR (Table V). These were involved in the endocrine system, development, and the circulatory, excretory, digestive, nervous and immune systems. Of these, the PPAR signaling pathway was one of the most associated pathways, and was classified and assigned to the functional group of the endocrine system. PPARs are ligand-activated TFs (members of the nuclear receptor family) which offer promising 
Table I. Significant pathways associated with DR in the functional class of cellular processes.

\begin{tabular}{|c|c|c|c|}
\hline Pathways & Map B & No. of genes & No. of TFs \\
\hline 04142: Lysosome & Transport and catabolism & 119 & 28 \\
\hline 04144: Endocytosis & Transport and catabolism & 199 & 22 \\
\hline 04145: Phagosome & Transport and catabolism & 140 & 22 \\
\hline 04530: Tight junction & Cell communication & 127 & 34 \\
\hline 04810: Regulation of actin cytoskeleton & Cell motility & 206 & 26 \\
\hline 05142: Chagas disease (American trypanosomiasis) & & 99 & 28 \\
\hline
\end{tabular}

DR, diabetic retinopathy; TFs, transcription factors.

Table II. Significant pathways associated with DR in the functional class of environmental and genetic information processing.

\begin{tabular}{|c|c|c|c|}
\hline Pathways & Map B & No. of genes & No. of TFs \\
\hline 02010: ABC transporters & Membrane transport & 44 & 25 \\
\hline 04512: ECM-receptor interaction & Signaling molecules and interaction & 83 & 34 \\
\hline 04012: ErbB signaling pathway & Signal transduction & 86 & 40 \\
\hline 04150: mTOR signaling pathway & Signal transduction & 52 & 30 \\
\hline 04370: VEGF signaling pathway & Signal transduction & 74 & 25 \\
\hline 04310: Wnt signaling pathway & Signal transduction & 145 & 30 \\
\hline 04070: Phosphatidylinositol signaling system & Signal transduction & 73 & 26 \\
\hline 04130: SNARE interactions in vesicular transport & Folding, sorting and degradation & 35 & 6 \\
\hline 03050: Proteasome & Folding, sorting and degradation & 44 & 20 \\
\hline 03440: Homologous recombination & Replication and repair & 27 & 17 \\
\hline 03030: DNA replication & Replication and repair & 35 & 23 \\
\hline 00970: Aminoacyl-tRNA biosynthesis & Translation & 41 & 7 \\
\hline 03010: Ribosome & Translation & 53 & 19 \\
\hline 03020: RNA polymerase ${ }^{a}$ & Transcription & 24 & 29 \\
\hline
\end{tabular}

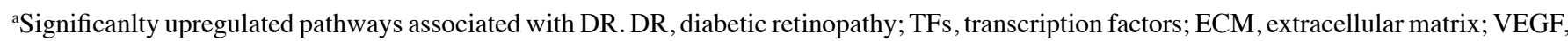
vascular endothelial growth factor; ABC, ATP-binding cassette; mTOR, mammalian target of rapamycin; SNARE, soluble N-ethylmaleimidesensitive attachment protein receptor.

Table III. Significant pathways associated with DR in the functional class of human diseases.

\begin{tabular}{|c|c|c|c|}
\hline Pathways & Map B & $\begin{array}{l}\text { No. of } \\
\text { genes }\end{array}$ & $\begin{array}{c}\text { No. of } \\
\text { TFs }\end{array}$ \\
\hline 04950: Maturity onset diabetes of the young & Endocrine and metabolic diseases & 25 & 25 \\
\hline 04940: Type I diabetes mellitus & Endocrine and metabolic diseases & 40 & 30 \\
\hline 05330: Allograft rejection & Immune diseases & 33 & 22 \\
\hline 05416: Viral myocarditis & Cardiovascular diseases & 65 & 21 \\
\hline 05410: Hypertrophic cardiomyopathy (HCM) & Cardiovascular diseases & 81 & 32 \\
\hline 05412: Arrhythmogenic right ventricular cardiomyopathy (ARVC) & Cardiovascular diseases & 73 & 43 \\
\hline 05100: Bacterial invasion of epithelial cells & Infectious diseases: bacterial & 66 & 29 \\
\hline 05140: Leishmaniasis & Infectious diseases: parasitic & 62 & 20 \\
\hline 05143: African trypanosomiasis & Infectious diseases: parasitic & 30 & 19 \\
\hline 05146: Amoebiasis & Infectious diseases: parasitic & 106 & 26 \\
\hline 05145: Toxoplasmosis & Infectious diseases: parasitic & 123 & 19 \\
\hline 05211: Renal cell carcinoma & Cancers & 70 & 30 \\
\hline 05212: Pancreatic cancer & Cancers & 69 & 25 \\
\hline
\end{tabular}

DR, diabetic retinopathy; TFs, transcription factors. 
Table IV. Significant pathways associated with DR in the functional class of metabolism.

\begin{tabular}{|c|c|c|c|}
\hline Pathways & Map B & $\begin{array}{l}\text { No. of } \\
\text { genes }\end{array}$ & $\begin{array}{c}\text { No. of } \\
\text { TFs }\end{array}$ \\
\hline 00010: Glycolysis/gluconeogenesis & Carbohydrate metabolism & 57 & 14 \\
\hline 00500: Starch and sucrose metabolism & Carbohydrate metabolism & 32 & 12 \\
\hline 00520: Amino sugar and nucleotide sugar metabolism & Carbohydrate metabolism & 48 & 20 \\
\hline 00620: Pyruvate metabolism & Carbohydrate metabolism & 39 & 22 \\
\hline 00030: Pentose phosphate pathway & Carbohydrate metabolism & 25 & 14 \\
\hline 00630: Glyoxylate and dicarboxylate metabolism & Carbohydrate metabolism & 18 & 30 \\
\hline 00250: Alanine, aspartate and glutamate metabolism & Amino acid metabolism & 32 & 22 \\
\hline 00290: Valine, leucine and isoleucine biosynthesis & Amino acid metabolism & 11 & 30 \\
\hline 00310: Lysine degradation & Amino acid metabolism & 43 & 26 \\
\hline 00230: Purine metabolism & Nucleotide metabolism & 152 & 24 \\
\hline 00590: Arachidonic acid metabolism & Lipid metabolism & 77 & 21 \\
\hline 00591: Linoleic acid metabolism & Lipid metabolism & 40 & 13 \\
\hline 00100: Steroid biosynthesis ${ }^{\mathrm{a}}$ & Lipid metabolism & 18 & 17 \\
\hline 00670: One carbon pool by folate & Metabolism of cofactors and vitamins & 17 & 26 \\
\hline 00790: Folate biosynthesis ${ }^{\mathrm{a}}$ & Metabolism of cofactors and vitamins & 11 & 28 \\
\hline 00900: Terpenoid backbone biosynthesis ${ }^{\mathrm{a}}$ & Metabolism of terpenoids and polyketides & 13 & 22 \\
\hline 00604: Glycosphingolipid biosynthesis - ganglio series ${ }^{\mathrm{a}}$ & Glycan biosynthesis and metabolism & 15 & 19 \\
\hline 00534: Glycosaminoglycan biosynthesis - heparan sulfate & Glycan biosynthesis and metabolism & 24 & 25 \\
\hline
\end{tabular}

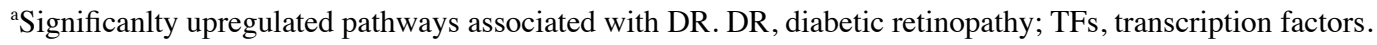

Table V. Significant pathways associated with DR in the functional class of organismal systems.

\begin{tabular}{|c|c|c|c|}
\hline Pathways & Map B & No. of genes & No. of TFs \\
\hline 03320: PPAR signaling pathway ${ }^{\mathrm{a}}$ & Endocrine system & 71 & 25 \\
\hline 04910: Insulin signaling pathway & Endocrine system & 128 & 27 \\
\hline 04916: Melanogenesis & Endocrine system & 95 & 40 \\
\hline 04920: Adipocytokine signaling pathway & Endocrine system & 67 & 19 \\
\hline 04320: Dorso-ventral axis formation & Development & 22 & 24 \\
\hline 04360: Axon guidance & Development & 129 & 28 \\
\hline 04380: Osteoclast differentiation & Development & 112 & 34 \\
\hline 04270: Vascular smooth muscle contraction & Circulatory system & 110 & 32 \\
\hline 04960: Aldosterone-regulated sodium reabsorption & Excretory system & 44 & 33 \\
\hline 04962: Vasopressin-regulated water reabsorption & Excretory system & 43 & 27 \\
\hline 04970: Salivary secretion & Digestive system & 70 & 41 \\
\hline 04972: Pancreatic secretion & Digestive system & 99 & 44 \\
\hline 04720: Long-term potentiation & Nervous system & 63 & 27 \\
\hline 04730: Long-term depression & Nervous system & 68 & 22 \\
\hline 04660: T cell receptor signaling pathway & Immune system & 106 & 33 \\
\hline 04664: Fc epsilon RI signaling pathway & Immune system & 77 & 28 \\
\hline 04666: Fc gamma R-mediated phagocytosis & Immune system & 86 & 39 \\
\hline 04670: Leukocyte transendothelial migration & Immune system & 115 & 26 \\
\hline
\end{tabular}

aSignificantly upregulated pathways associated with DR. DR, diabetic retinopathy; TFs, transcription factors; PPAR, peroxisome proliferatoractivated receptor.

targets for the development of novel, efficient treatments for several metabolic disorders. An indication suggesting that antidiabetic thiazolidinediones and adipogenic prostanoids are ligands of one of the PPARs reveals a novel signaling pathway 
that directly links these compounds to processes involved in glucose homeostasis and lipid metabolism, including adipocyte differentiation (35).

Candidate TF selection associated with DR. To predict TFs potentially involved in the regulation of DR, we performed an analysis of TFBSs and predicted TFs using the significant genes in each identified pathway. Based on the cut-off value of TF importance, we identified the candidate TFs related to DR with potential target genes which are co-regulated in each of the above 69 pathways. The details are available upon request. As a result, 2 protein families, PPARs and SMADs, including members PPAR $\alpha$, PPAR $\gamma$, PPAR_DR1, SMAD, SMAD3 and SMAD4, were predicted as candidate TFs in the majority of the identified pathways, particularly the downregulated pathways. PPARs are ligand-activated nuclear TFs that control gene expression by binding to specific response elements (PPREs) within promoters. They play a critical physiological role as lipid sensors and regulators of lipid metabolism (36). More potent synthetic PPAR ligands, including the fibrates and thiazolidinediones, have proven effective in the treatment of dyslipidemia and diabetes (35). The powerful therapeutic ability of PPAR $\alpha$ and PPAR $\gamma$ agonists to favorably influence systemic lipid levels, glucose homeostasis, and the risk of atherosclerosis (in the case of PPAR $\alpha$ activation in humans) have been demonstrated (37). PPAR $\gamma$ plays a vitally important role in the pathogenesis of DR by inhibiting retinal leukostasis and leakage in response to diabetes (38). Fenofibrate, a PPAR $\alpha$ agonist, has demonstrated robust protective effects against DR in diabetic patients (39). Our data also support the hypothesis that PPAR $\alpha$ and PPAR $\gamma$ may be important therapeutic targets for the management of DR.

The TGF- $\beta$ signal is predominantly transduced by a family of TFs, the Smad proteins (40). After binding the TGF- $\beta$ ligand from outside the cell surface, the type II receptor activates the type I receptor kinase, and this is followed by the phosphorylation of receptor-regulated Smads (R-Smads), Smad 2 and Smad3. After associating with a common-partner Smad (co-Smad), or Smad4, the Smad complex translocates to the nucleus where it regulates the expression of target genes $(40,41)$. TGF- $\beta$ is a multifunctional cytokine with a number of biological effects, such as cell growth, differentiation and immunomodulation (42). A recent study found that the expression of TGF- $\beta$ and Smad4 was increased in the retinal neovascular membrane of mice with oxygen-induced retinopathy (43). A previous study also indicated that increased Smad2/3 phosphorylation levels and increased TGF- $\beta$ signaling do in fact occur in the retinal vessels of diabetic rats, and the concordant attenuation of such signaling by drugs which protect the vessels from the effects of diabetes through different mechanisms suggests that the increased signaling contributes to the vascular pathology (44).

In conclusion, we applied GSEA to re-analyze the published microarray datasets of DR and performed candidate TF selection. Finally, we identified 10 upregulated pathways, including the type I diabetes mellitus and PPAR signaling pathways, as well as 59 downregulated pathways, including the ErbB signaling pathway and the mTOR signaling pathway. Furthermore, co-expression networks of related pathways were constructed using the significant core genes and TFs, such as PPARG and SMAD4. These may be helpful to systematically understand the molecular mechanisms of diabetic retinopathy in genome-wide.

\section{Acknowledgements}

We acknowledge financial support from the Scientific Research Foundation and Academic and Technology Leaders Introduction Project, the 211 Project of Anhui University (grant nos. 10117700023 and 02303203-32030081), the Student Research Training Program of Anhui University (grant no. J18520131), the Natural Science Foundation Project of Anhui (grant nos. 1508085MH189 and 1508085QC63) and the Education Revitalization Project of Anhui: Stem Cell and Translational Medicine (grant no. Y05201374).

\section{References}

1. Klein R, Klein BE, Moss SE and Cruickshanks KJ: The Wisconsin Epidemiologic Study of Diabetic Retinopathy: XVII. The 14-year incidence and progression of diabetic retinopathy and associated risk factors in type 1 diabetes. Ophthalmology 105: 1801-1815, 1998.

2. Wong TY, Klein R, Islam FM, Cotch MF, Folsom AR, Klein BE, Sharrett AR and Shea S: Diabetic retinopathy in a multi-ethnic cohort in the United States. Am J Ophthalmol 141: 446-455, 2006.

3. Ng DP: Human genetics of diabetic retinopathy: Ccurrent perspectives. J Ophthalmol 2010: 172593, 2010.

4. Mohamed Q, Gillies MC and Wong TY: Management of diabetic retinopathy: Aa systematic review. JAMA 298: 902-916, 2007.

5. Todd JA, Walker NM, Cooper JD, Smyth DJ, Downes K, Plagnol V, Bailey R, Nejentsev S, Field SF, Payne F, et al; Genetics of Type 1 Diabetes in Finland; Wellcome Trust Case Control Consortium: Robust associations of four new chromosome regions from genome-wide analyses of type 1 diabetes. Nat Genet 39: 857-864, 2007.

6. Brucklacher RM, Patel KM, VanGuilder HD, Bixler GV, Barber AJ, Antonetti DA, Lin CM, LaNoue KF, Gardner TW, Bronson SK and Freeman WM: Whole genome assessment of the retinal response to diabetes reveals a progressive neurovascular inflammatory response. BMC Med Genomics 1: 26, 2008

7. Costa V, Gallo MA, Letizia F, Aprile M, Casamassimi A and Ciccodicola A: PPARG: Gene expression regulation and next-generation sequencing for unsolved issues. PPAR Res 2010: 409168, 2010.

8. Lorenzi $\mathrm{M}$ and Gerhardinger C: Early cellular and molecular changes induced by diabetes in the retina. Diabetologia 44 : 791-804, 2001.

9. Warpeha KM and Chakravarthy U: Molecular genetics of microvascular disease in diabetic retinopathy. Eye (Lond) 17: 305-311, 2003.

10. Ray D, Mishra M, Ralph S, Read I, Davies R and Brenchley P: Association of the VEGF gene with proliferative diabetic retinopathy but not proteinuria in diabetes. Diabetes 53: 861-864, 2004.

11. Kumaramanickavel G, Ramprasad VL, Sripriya S, Upadyay NK, Paul PG and Sharma T: Association of Gly82Ser polymorphism in the RAGE gene with diabetic retinopathy in type II diabetic Asian Indian patients. J Diabetes Complications 16: 391-394, 2002.

12. Awata T, Inoue K, Kurihara S, Ohkubo T, Watanabe M, Inukai K, Inoue I and Katayama S: A common polymorphism in the 5'-untranslated region of the VEGF gene is associated with diabetic retinopathy in type 2 diabetes. Diabetes 51: 1635-1639, 2002.

13. Wang Y, Ng MC, Lee SC, So WY, Tong PC, Cockram CS, Critchley JA and Chan JC: Phenotypic heterogeneity and associations of two aldose reductase gene polymorphisms with nephropathy and retinopathy in type 2 diabetes. Diabetes Care 26: 2410-2415, 2003.

14. Suganthalakshmi B, Anand R, Kim R, Mahalakshmi R, Karthikprakash S, Namperumalsamy $P$ and Sundaresan $P$ : Association of VEGF and eNOS gene polymorphisms in type 2 diabetic retinopathy. Mol Vis 12: 336-341, 2006.

15. Beránek M, Kanková K, Benes P, Izakovicová-Hollá L, Znojil V, Hájek D, Vlková E and Vácha J: Polymorphism R25P in the gene encoding transforming growth factor-beta (TGF-beta1) is a newly identified risk factor for proliferative diabetic retinopathy. Am J Med Genet 109: 278-283, 2002.

16. Lam HC, Lee JK, Lu CC, Chu CH, Chuang MJ and Wang MC: Role of endothelin in diabetic retinopathy. Curr Vasc Pharmacol 1: 243-250, 2003. 
17. Santos KG, Tschiedel B, Schneider J, Souto K and Roisenberg I: Diabetic retinopathy in Euro-Brazilian type 2 diabetic patients: relationship with polymorphisms in the aldose reductase, the plasminogen activator inhibitor-1 and the methylenetetrahydrofolate reductase genes. Diabetes Res Clin Pract 61: 133-136, 2003.

18. Amano S, Yamagishi S, Koda Y, Tsuneoka M, Soejima M, Okamoto T, Inagaki Y, Yamada K and Kimura H: Polymorphisms of sorbitol dehydrogenase ( $\mathrm{SDH}$ ) gene and susceptibility to diabetic retinopathy. Med Hypotheses 60: 550-551, 2003.

19. Yoshioka K, Yoshida T, Takakura Y, Umekawa T, Kogure A, Toda $\mathrm{H}$ and Yoshikawa T: Relation between polymorphisms G1704T and G82S of rage gene and diabetic retinopathy in Japanese type 2 diabetic patients. Intern Med 44: 417-421, 2005.

20. Edgar R, Domrachev M and Lash AE: Gene Expression Omnibus: NCBI gene expression and hybridization array data repository. Nucleic Acids Res 30: 207-210, 2002.

21. Barrett T, Wilhite SE, Ledoux P, Evangelista C, Kim IF, Tomashevsky M, Marshall KA, Phillippy KH, Sherman PM, Holko M, et al: NCBI GEO: Aarchive for functional genomics data set - update. Nucleic Acids Res 41: D991-D995, 2013.

22. Subramanian A, Tamayo P, Mootha VK, Mukherjee S, Ebert BL, Gillette MA, Paulovich A, Pomeroy SL, Golub TR, LanderES, et al: Gene set enrichment analysis: a knowledge-based approach for interpreting genome-wide expression profiles. Proc Natl Acad Sci USA 102: 15545-15550, 2005.

23. He K, Chen Z, Ma Y and Pan Y: Identification of high-copperresponsive target pathways in Atp7b knockout mouse liver by GSEA on microarray data sets. Mamm Genome 22: 703-713, 2011.

24. Zhao H, Wang Q, Bai C, He K and Pan Y: A cross-study gene set enrichment analysis identifies critical pathways in endometriosis. Reprod Biol Endocrinol 7: 94, 2009.

25. Gautier L, Cope L, Bolstad BM and Irizarry RA: affy - analysis of Affymetrix GeneChip data at the probe level. Bioinformatics 20: 307-315, 2004.

26. Chiaretti S, Li X, Gentleman R, Vitale A, Vignetti M, Mandelli F, Ritz J and Foa R: Gene expression profile of adult T-cell acute lymphocytic leukemia identifies distinct subsets of patients with different response to therapy and survival. Blood 103: 2771-2778, 2004.

27. Gotea V and Ovcharenko I: DiRE: Iidentifying distant regulatory elements of co-expressed genes. Nucleic Acids Res 36: W133-W139, 2008.

28. Ma JH, Li J, Wang JJ and Zhang SX: X-box binding protein 1 (XBP1) is a crucial regulator of endothelial tight junction and protects the blood-retinal barrier in diabetic retinopathy. Invest Ophthalmol Vis Sci 55: 2253, 2014.

29. Akhtar S, Almubrad T, Bron AJ, Yousif MH, Benter IF and Akhtar S: Role of epidermal growth factor receptor (EGFR) in corneal remodelling in diabetes. Acta Ophthalmol 87: 881-889, 2009.
30. McClintock JL and Ceresa BP: Transforming growth factor\{alpha\} enhances corneal epithelial cell migration by promoting EGFR recycling. Invest Ophthalmol Vis Sci 51: 3455-3461, 2010.

31. Humar R, Kiefer FN, Berns H, Resink TJ and Battegay EJ: Hypoxia enhances vascular cell proliferation and angiogenesis in vitro via rapamycin (mTOR)-dependent signaling. FASEB J 16: 771-780, 2002.

32. Cai J, Ahmad S, Jiang WG, Huang J, Kontos CD, Boulton M and Ahmed A: Activation of vascular endothelial growth factor receptor-1 sustains angiogenesis and $\mathrm{Bcl}-2$ expression via the phosphatidylinositol 3-kinase pathway in endothelial cells. Diabetes 52: 2959-2968, 2003.

33. Klein R, Lee KE, Gangnon RE and Klein BE: The 25-year incidence of visual impairment in type 1 diabetes mellitus the wisconsin epidemiologic study of diabetic retinopathy. Ophthalmology 117: 63-70, 2010.

34. Wang QJ: PKD at the crossroads of DAG and PKC signaling. Trends Pharmacol Sci 27: 317-323, 2006.

35. Lemberger T, Desvergne B and Wahli W: Peroxisome proliferator-activated receptors: a nuclear receptor signaling pathway in lipid physiology. Annu Rev Cell Dev Biol 12: 335-363, 1996.

36. Hihi AK, Michalik L and Wahli W: PPARs: Transcriptional effectors of fatty acids and their derivatives. Cell Mol Life Sci 59: 790-798, 2002

37. Berger J and Moller DE: The mechanisms of action of PPARs. Annu Rev Med 53: 409-435, 2002.

38. Song MK, Roufogalis BD and Huang TH: Modulation of diabetic retinopathy pathophysiology by natural medicines through PPAR- $\gamma$-related pharmacology. Br J Pharmacol 165: 4-19, 2012.

39. Chen Y, Hu Y, Lin M, Jenkins AJ, Keech AC, Mott R, Lyons TJ and Ma JX: Therapeutic effects of PPAR $\alpha$ agonists on diabetic retinopathy in type 1 diabetes models. Diabetes 62: 261-272, 2013.

40. Massagué $\mathrm{J}$ and Wotton D: Transcriptional control by the TGF- $\beta$ /Smad signaling system. EMBO J 19: 1745-1754, 2000.

41. Derynck R and Zhang YE: Smad-dependent and Smadindependent pathways in TGF- $\beta$ family signalling. Nature 425 : 577-584, 2003.

42. Kim ES, Kim MS and Moon A: TGF-beta-induced upregulation of MMP-2 and MMP-9 depends on p38 MAPK, but not ERK signaling in MCF10A human breast epithelial cells. Int J Oncol 25: 1375-1382, 2004.

43. Yingchuan F, Chuntao L, Hui $\mathrm{C}$ and Jianbin $\mathrm{H}$ : Increased expression of TGF- $\beta 1$ and Smad 4 on oxygen-induced retinopathy in neonatal mice. In: Retinal Degenerative Diseases. Springer-Verlag, New York, NY, pp71-77, 2010.

44. Gerhardinger C, Dagher Z, Sebastiani P, Park YS and Lorenzi M: The transforming growth factor- $\beta$ pathway is a common target of drugs that prevent experimental diabetic retinopathy. Diabetes 58: 1659-1667, 2009. 